\title{
Sphingosine kinase 1 participates in insulin signalling and regulates glucose metabolism and homeostasis in KK/Ay diabetic mice
}

\author{
M. M. Ma • J. L. Chen • G. G. Wang • H. Wang • Y. Lu • \\ J. F. Li • J. Yi • Y. J. Yuan • Q. W. Zhang • J. Mi • \\ L. Sh. Wang • H. F. Duan • C. T. Wu
}

Received: 5 November 2006 / Accepted: 15 December 2006/ Published online: 31 January 2007

(C) Springer-Verlag 2007

\begin{abstract}
Aims/hypothesis The aim of this study was to determine the potential role of sphingosine kinase 1 (SPHK1), a key sphingolipid metabolic enzyme, in glucose metabolism and homeostasis.

Methods SMMC-7721 hepatoma cells and C2C12 myotube cells were used to explore the role of SPHK1 in glucose uptake in vitro. KK/Ay type 2 diabetic mice, which were transfected with adenovirus harbouring the human SPHK1 gene by i.v. injection, were used to investigate the glucoselowering effects of SPHK1 in vivo.
\end{abstract}

M. M. Ma and J. L. Chen have contributed equally to this work.

Electronic supplementary material The online version of this article (doi:10.1007/s00125-006-0589-5) contains supplementary material, which is available to authorised users.

M. M. Ma • Y. J. Yuan

School of Chemical Engineering and Technology,

Tianjin University,

Tianjin, People's Republic of China

J. L. Chen · G. G. Wang • J. F. Li • Q. W. Zhang • J. Mi •

L. S. Wang $\cdot$ H. F. Duan $(\bowtie) \cdot$ C. T. Wu $(\bowtie)$

Department of Experimental Haematology,

Beijing Institute of Radiation Medicine,

Beijing, People's Republic of China

e-mail: duanhf0720@yahoo.com.cn

e-mail: wuct@nic.bmi.ac.cn

H. Wang Y. Lu

Affiliated Hospital, Academy of Military Medical Sciences,

Beijing, People's Republic of China

J. Yi

Department of Cardiac Medicine, General Hospital of PLA,

Beijing, People's Republic of China
Results The basal glucose uptake and the insulin-stimulated glucose uptake in both 7721 cells and $\mathrm{C} 2 \mathrm{C} 12$ cells were markedly enhanced when SPHK1 was overexpressed by adenovirus-mediated gene transfer, whereas they were substantially reduced when the expression of SPHK1 was inhibited or the activity of SPHK1 was blocked. Insulin could activate SPHK1 of both cell lines in a dosedependent manner. SPHK1 gene delivery significantly reduced the blood glucose level of KK/Ay diabetic mice, but had no effect on that of normal animals. It also attenuated elevated levels of plasma insulin, NEFA, triacylglycerol, cholesterol and LDL, significantly ameliorated hyperglycaemia-induced injury of liver, heart and kidney, and enhanced phosphorylation of insulin-signalling kinases such as Akt and glycogen synthase kinase $3 \beta$ in livers of the diabetic animals.

Conclusions/interpretation SPHK1 is involved in insulin signalling and plays an important role in the regulation of glucose and fat metabolism; adenovirus-mediated SPHKI gene transfer might provide a novel strategy in the treatment of type 2 diabetes mellitus.

Keywords Glucose metabolism · Insulin signalling ·

Sphingosine kinase $1 \cdot$ Type 2 diabetes mellitus

$\begin{array}{ll}\text { Abbreviations } \\ \text { Ad-GFP } & \begin{array}{l}\text { adenoviral vectors containing green } \\ \text { fluorescent protein gene } \\ \text { adenoviral vectors containing } \\ \text { Ad-SPHK1 }\end{array} \\ & \begin{array}{l}\text { FLAG-labelled human sphingosine } \\ \text { kinase 1 gene }\end{array} \\ \text { alanine aminotransferase } \\ \text { AST } & \begin{array}{l}\text { aspartate aminotransferase } \\ \text { ceramide }\end{array}\end{array}$




$\begin{array}{ll}\text { CK } & \text { creatine kinase } \\ \text { DMS } & N, N \text {-dimethylsphingosine } \\ \text { GSK3B } & \text { glycogen synthase kinase } 3 \beta \\ \text { 2-D }\left[{ }^{3} \mathrm{H}\right] \mathrm{OG} & \text { 2-deoxy- }\left[{ }^{3} \mathrm{H}\right] \text { glucose } \\ \text { LDH } & \text { lactate dehydrogenase } \\ \text { PI3K } & \text { phosphatidylinositol 3-kinase } \\ \text { S1P } & \text { sphingosine 1-phosphate } \\ \text { siRNA } & \text { small interfering RNA } \\ \text { SP } & \text { sphingosine } \\ \text { SPHK1 } & \text { sphingosine kinase 1 }\end{array}$

\section{Introduction}

Type 2 diabetes mellitus is a progressive metabolic disorder developing from both environmental and undefined genetic factors, involving defects in all major organs controlling metabolism, such as liver, skeletal muscle, adipose tissue and beta cells. Although the primary factors causing this disease remain unknown, it is clear that insulin resistance plays an important role in the pathogenesis, development and outcome of type 2 diabetes mellitus [1]. Overloading of nutrients to peripheral tissues can lead to the formation of metabolites, such as NEFAs, or to the production of a chronic inflammatory state characterised by elevated circulating levels of inflammatory mediators, such as TNF- $\alpha$ [2-7]. These metabolites and mediators are regarded as the major cause of insulin resistance. It has been documented that ceramide (CER), one of the sphingolipid metabolites, is a key intermediate linking certain nutrients (e.g. NEFAs) and inflammatory cytokines (e.g. TNF- $\alpha$ ) with insulin resistance [8-14].

It is well established that the metabolism of lipid and glucose are closely correlated. As the two main classes of substance associated with energetic metabolism, lipid and glucose can transform reciprocally in vivo. The metabolites of lipid can influence glucose metabolism, or vice versa. For example, NEFAs and CER play important roles in the development of insulin resistance, and even diabetes mellitus, and phosphatidylinositol 3-kinase (PI3K), which is an important lipid kinase in lipid metabolism, has also been proved to be a key component in the insulin-signalling pathway, and thereby plays a vital role in the control of glucose metabolism [15-17]. Sphingolipids are a class of enigmatic lipids and their metabolism leads to the generation of several bioactive products including CER, sphingosine (SP) and sphingosine1-phosphate (S1P) [18, 19], which are important signalling molecules participating in a variety of important mammalian cell processes including proliferation, differentiation, mobility and apoptosis. More interestingly, the catabolic pathway of sphingolipids is complex with several potential points of regulation and modulation [19].
For example, the dynamic balance between cellular concentrations of CER/SP and S1P determines whether cells survive or die, which is termed 'sphingolipid rheostat'. Generally, CER and SP, the precursor of S1P, are associated with cell growth arrest and apoptosis, whereas S1P has been implicated in cell proliferation and survival [20-24].

Sphingosine kinase 1 (SPHK1) is a key enzyme in the sphingolipid metabolic pathway and acts as an essential checkpoint in regulating the relative levels of CER/SP and S1P [21]. Moreover, SPHK1 is similar to the abovementioned PI3K in several aspects: (1) both of them are highly conserved lipid kinases catalysing lipid phosphorylation in phospholipid metabolic pathways; (2) the catalysed products of both kinases act as the second messenger of cellular signalling; and (3) the increase of activity of both kinases is closely related to cell survival and proliferation. Therefore, it is of interest to know whether SPHK1 would be another important lipid kinase involved in glucose metabolism and whether gene transfer of this factor could greatly improve glucose homeostasis in diabetic mice. To answer these questions, we explored the role of SPHK1 in glucose uptake and insulin sensitivity in vitro and further examined the metabolic effects of SPHK1 in vivo by gene delivery using the KK/Ay type 2 diabetic mouse model.

\section{Materials and methods}

Materials Antibodies to Akt, Ser473-phosphorylated Akt, glycogen synthase kinase $3 \beta$ (GSK3B, previously known as GSK-3 $\beta$ ) and Ser9-phosphorylated GSK-3B were purchased from Cell Signaling Technology, Beverly, MA, USA. The adenovirus expression vector kit (AdEasy system) was purchased from Stratagene, La Jolla, CA, USA. S1P, SP, $N$, $N$-dimethylsphingosine (DMS) and 2-deoxy- $\left[{ }^{3} \mathrm{H}\right]$ glucose $\left(2-\mathrm{D}\left[{ }^{3} \mathrm{H}\right] \mathrm{OG}\right)$ were from Sigma, St. Louis, MO, USA. Lipofectamine 2000 reagent was the product of Invitrogen Life Technologies, Carlsbad, CA, USA.

Preparation of replication-deficient adenoviral vectors $A d-$ SPHK1 and Ad-GFP Replication-deficient (E1, E3 deleted) adenoviral vectors containing FLAG-labelled human sphingosine kinase 1 (Ad-SPHK1) were prepared by using the pAdEasy-1 system following the manufacturer's instructions. Replication-deficient adenovirus containing green fluorescent protein ( $\mathrm{Ad}-G F P$ ) was similarly obtained as a control. Recombinant adenoviruses were produced in HEK293 cells and then purified by $\mathrm{CsCl}$ gradient centrifugation and the final plaque-forming units were determined by titration on 293 cells under an agarose overlay.

Cell culture and treatment SMMC-7721 hepatoma cells and $\mathrm{C} 2 \mathrm{C} 12$ myotube cells were routinely cultured in 
DMEM supplemented with $10 \%$ fetal calf plasma and antibiotics (penicillin $100 \mathrm{IU} / \mathrm{ml}$, streptomycin $100 \mu \mathrm{g} / \mathrm{ml}$ ) in an atmosphere of $5 \% \mathrm{CO}_{2}$ at $37^{\circ} \mathrm{C}$. The cells were infected with $\mathrm{Ad}-S P H K 1$ or $\mathrm{Ad}-G F P$ as a control at a 100 multiplicity of infection for $24 \mathrm{~h}$, and then the cellular SPHK1 activity and extracellular S1P levels were measured.

$\left.2-D{ }^{3} H\right] O G$ uptake in vitro Cells were seeded in 48 -well plates, and $2-\mathrm{D}\left[{ }^{3} \mathrm{H}\right] \mathrm{OG}$ uptake assays were performed as described previously [25]. Briefly, after being incubated in serum-free DMEM overnight at $37^{\circ} \mathrm{C}$, the cells were infected with a 100 multiplicity of infection of Ad-SPHK1 for $24 \mathrm{~h}$ or stimulated with $0.1,0.5,2.5 \mu \mathrm{mol} / 1 \mathrm{~S} 1 \mathrm{P}$ for $30 \mathrm{~min}$ and then incubated with $100 \mathrm{nmol} / \mathrm{l}$ insulin for $15 \mathrm{~min}$. Glucose uptake was initiated by incubation with 2$\mathrm{D}\left[{ }^{3} \mathrm{H}\right] \mathrm{OG}$ at $18.5 \mathrm{kBq} / \mathrm{ml}$ for $10 \mathrm{~min}$ at $37^{\circ} \mathrm{C}$.

Measurement of SPHK1 activity Cells were scraped into buffer A $(0.1 \mathrm{~mol} / \mathrm{l}$ Tris- $\mathrm{HCl}[\mathrm{pH} 7.4]$ containing $20 \%[\mathrm{v} / \mathrm{v}]$ glycerol, $1 \mathrm{mmol} / 1$ mercaptoethanol, $1 \mathrm{mmol} / 1$ EDTA, $1 \mathrm{mmol} / 1 \mathrm{Na}_{3} \mathrm{VO}_{4}, 15 \mathrm{mmol} / \mathrm{l} \mathrm{NaF}, 10 \mu \mathrm{g} / \mathrm{ml}$ leupeptin and aprotinin, $1 \mathrm{mmol} / \mathrm{l}$ phenylmethylsulfonylfluoride and $0.5 \mathrm{mmol} / 1$ 4-deoxypyridoxine). Cells were lysed by freezing-thawing three times, and cytosolic fractions were prepared by centrifugation at $13,000 \times g$ for $20 \mathrm{~min}$. Tissue homogenates were prepared by homogenising the tissues in buffer A. SPHK1 activity was measured as previously described [26].

Assay of SIP levels The assay utilised an alkaline lipid extraction to selectively separate S1P from cell extracts or tissues. Extracted S1P was efficiently converted to SP by alkaline phosphatase treatment. SP thus formed was then quantitatively phosphorylated to ${ }^{32} \mathrm{P}$-labelled S1P using recombinant sphingosine kinase and $\gamma-{ }^{32} \mathrm{P}$-labelled ATP. Then S1P levels were measured as described previously [27].

Small interfering RNA treatment Inhibition of SPHK1 expression in 7721 and $\mathrm{C} 2 \mathrm{C} 12$ cell lines with SPHK1specific small interfering RNA (siRNA) was performed as described previously [28]. Briefly, 7721 or $\mathrm{C} 2 \mathrm{C} 12$ cells were seeded into six-well plates 1 day before the experiment. Scrambled siRNA (5'-AAT TCT CCG AAC GTG TCA CGT dTdT-3' and 5'-ACG TGA CAC GTT CGG AGA A dTdT-3') and SPHK1-specific siRNA (5'-GAG CUG CAA GGC CUU GCC C dTdT-3' and 5'-GGG CAA GGC CUU GCA GCU C dTdT-3') were transfected into the cells using Lipofectamine 2000 reagent. siRNA oligonucleotides were synthesised and annealed by a commercial supplier (SigmaProligo, The Woodlands, TX, USA).

Western blot analysis Tissue extract of livers was subjected to western blot analyses for phosphorylated and total Akt and GSK3B. All blots were immunoreacted with a primary antibody overnight at $4^{\circ} \mathrm{C}$. An ECL system (Amersham Biosciences, Amersham, Bucks, UK) was used to detect signal following the manufacturer's instructions.

Animals and treatment Female and male KK/Ay mice (12-14 weeks old) or C57BL/6 mice (6-8 weeks old) were obtained from the Institute of Experimental Animals, Chinese Academy of Medical Sciences. All animals were kept individually in plastic cage at $25^{\circ} \mathrm{C}$ with illumination for $12 \mathrm{~h}$, and the KK/Ay mice were given a high-fat diet and with water freely available. Animals were injected i.v. with $2 \times 10^{9}$ plaque-forming units of either Ad-SPHK1 or control Ad-GFP. Each group consisted of ten animals. Blood glucose levels were monitored weekly by the glucocard test (Arkray, Shanghai, Japan). Cardiac and liver functions were evaluated 21 days after gene delivery, and tissues were then processed for biochemical and morphological analyses. All experimental procedures were approved and carried out in compliance with the guidelines of the Institute of Radiation Medicine on the use of animals.

Metabolic measurements The levels of serum glucose, insulin, triacylglycerol, NEFA, cholesterol, HDL, LDL, creatine kinase (CK), lactate dehydrogenase (LDH), aspartate aminotransferase (AST) and alanine aminotransferase (ALT) and of liver glycogen and triacylglycerol were measured as previously described [29].

Glucose tolerance test The glucose tolerance test was performed after fasting for $16 \mathrm{~h}$. Glucose $(2 \mathrm{~g} / \mathrm{kg})$ was administered orally, and blood was collected from the tail vein at $0,30,60$ and $120 \mathrm{~min}$ for measurement of serum glucose.

Histological analysis Samples of heart and liver were fixed in 10\% neutral-buffered formalin, embedded in paraffin, cut and stained with haematoxylin and eosin, and then analysed microscopically and morphometrically. Furthermore, the ultrastructures of heart and kidney of diabetic mice were assessed by electron microscopy, and the morphological features of cardiac tissues in each group were quantified as described previously [30].

Statistical analysis Data are expressed as means \pm SEM, and $n$ indicates the number of experiments. Paired Student's $t$ tests were used for comparison between two groups. For multiple comparisons, results were analysed by factorial ANOVA using Statistical Analysis System software (SAS8.00). A value of $p<0.05$ was considered statistically significant. 


\section{Results}

Effects of SPHK1 on glucose uptake and insulin-induced glucose uptake in vitro We first explored the role of SPHK1 in glucose uptake and insulin sensitivity in vitro. Ad-SPHK1 infection resulted in a marked increase (about fourfold) in SPHK1 activity in both 7721 and C2C12 cells (Electronic supplementary material [ESM] Fig. 1a), which led to an obvious increase in extracellular S1P levels (ESM Fig. 1b) and the basal and insulin-stimulated uptake of glucose by both cell lines as well (Fig. 1a). Blocking the activity of SPHK1 by DMS, a potent inhibitor of SPHK1, or inhibiting the expression of SPHK1 by siRNA caused a significant decrease in both the basal and insulin-induced glucose uptake (Fig. 1b,c). S1P also enhanced the basal and insulin-induced uptake of glucose by both cells in a dosedependent manner (ESM Fig. 2). These results show that SPHK1 plays an important role in glucose uptake. We then determined whether SPHK1 is implicated in insulin signalling. Results showed that insulin could enhance the
SPHK1 activity in both 7721 and $\mathrm{C} 2 \mathrm{C} 12$ cell lines in a dose-dependent manner (Fig. 1d), indicating that SPHK1 is involved in insulin signalling.

Effects of SPHK1 gene delivery on serum glucose and lipid profiles in $K K / A y$ diabetic mice Ad-SPHK1 was injected i.v. into $\mathrm{KK} / \mathrm{Ay}$ diabetic mice to determine glucoselowering effects of SPHK1 in vivo. The mice injected with Ad-SPHK1 showed a dramatic reduction in blood glucose levels, which lasted for at least 3 weeks. However, the diabetic mice injected with Ad-GFP showed no significant change in blood glucose level (Fig. 2a). Oral glucose tolerance tests showed that SPHK1 gene transfer resulted in an improved glucose tolerance with lower glucose level at all the time points after glucose loading (Fig. 2b). High serum insulin level, which is a result of insulin resistance, was markedly lowered by administration of Ad-SPHK1 (Fig. 2c) and high serum NEFA, which is a major cause of insulin resistance, was substantially reduced by Ad-SPHK1 injection (Fig. 2d). The effect of SPHK1 on blood fat
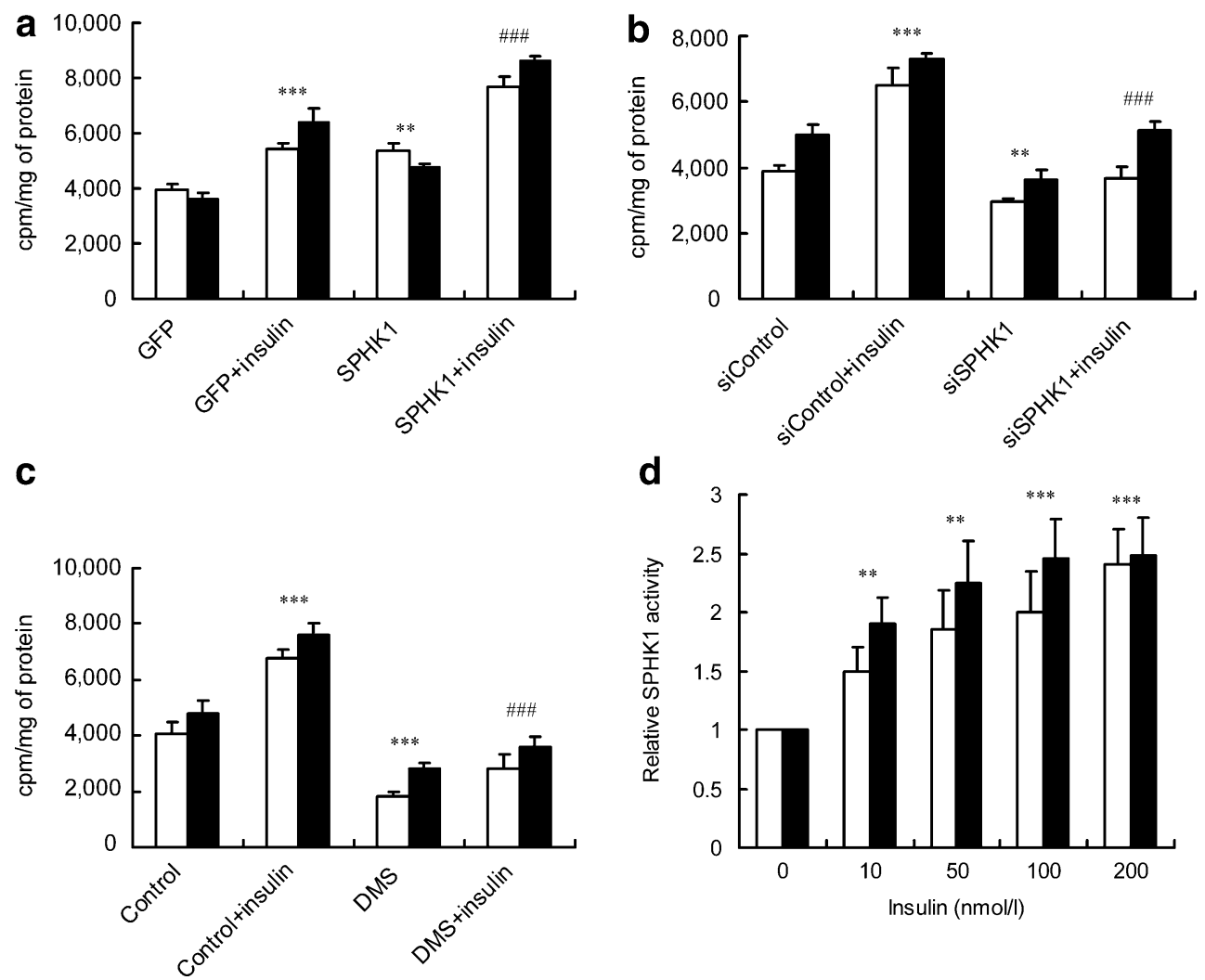

Fig. 1 SPHK1/S1P participates in insulin signalling and enhances cell glucose uptake. a Ad-SPHK1 infection enhances the basal and insulinstimulated uptake of glucose by 7721 and $\mathrm{C} 2 \mathrm{C} 12$ cell lines. The cells were infected with Ad-SPHK1 for $24 \mathrm{~h}$, and then the uptake of glucose by cells was determined. b, c Inhibition of SPHK1 expression by RNA interference (si) or blockage of SPHK1 activity by DMS reduced the basal and insulin-induced uptake of glucose by both 7721 and $\mathrm{C} 2 \mathrm{C} 12$

cells. Three independent experiments were performed, producing similar results. Results are shown as means \pm SEM of triplicates of one

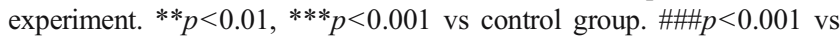
plus insulin control group. d Insulin enhances SPHK1 activity in both $\mathrm{C} 2 \mathrm{C} 12$ and 7721 cells in a dose-dependent manner (indicated concentrations of insulin for $2 \mathrm{~h}$ ). Data are means \pm SEM $(n=3)$. $* * p<0.01$, *** $p<0.001$ vs control. Black bars 7721 cells, open bars C2C12 cells 
Fig. 2 SPHK1 gene delivery reduces blood glucose and improves the plasma lipid profiles in KK/Ay diabetic mice. a Blood glucose concentrations of KK/Ay mice injected i.v. with adenovirus encoding GFP or SPHK1 at indicated times after adenovirus injection. b Blood glucose concentration during oral glucose tolerance tests of KK/Ay mice injected with adenovirus encoding GFP or SPHK1. Squares SPHK1, diamonds GFP. c Serum insulin concentration of KK mice injected with $\mathrm{Ad}-G F P$ or Ad-SPHK1 at the endpoint of the study. d Triacylglycerol $(T G)$, cholesterol (CHO), HDL and LDL levels. Open bars GFP, black bars SPHK1. e NEFA levels. Data are means \pm SEM $(n=10) . * p<0.05$, $* * p<0.01, * * * p<0.001$ vs GFP group
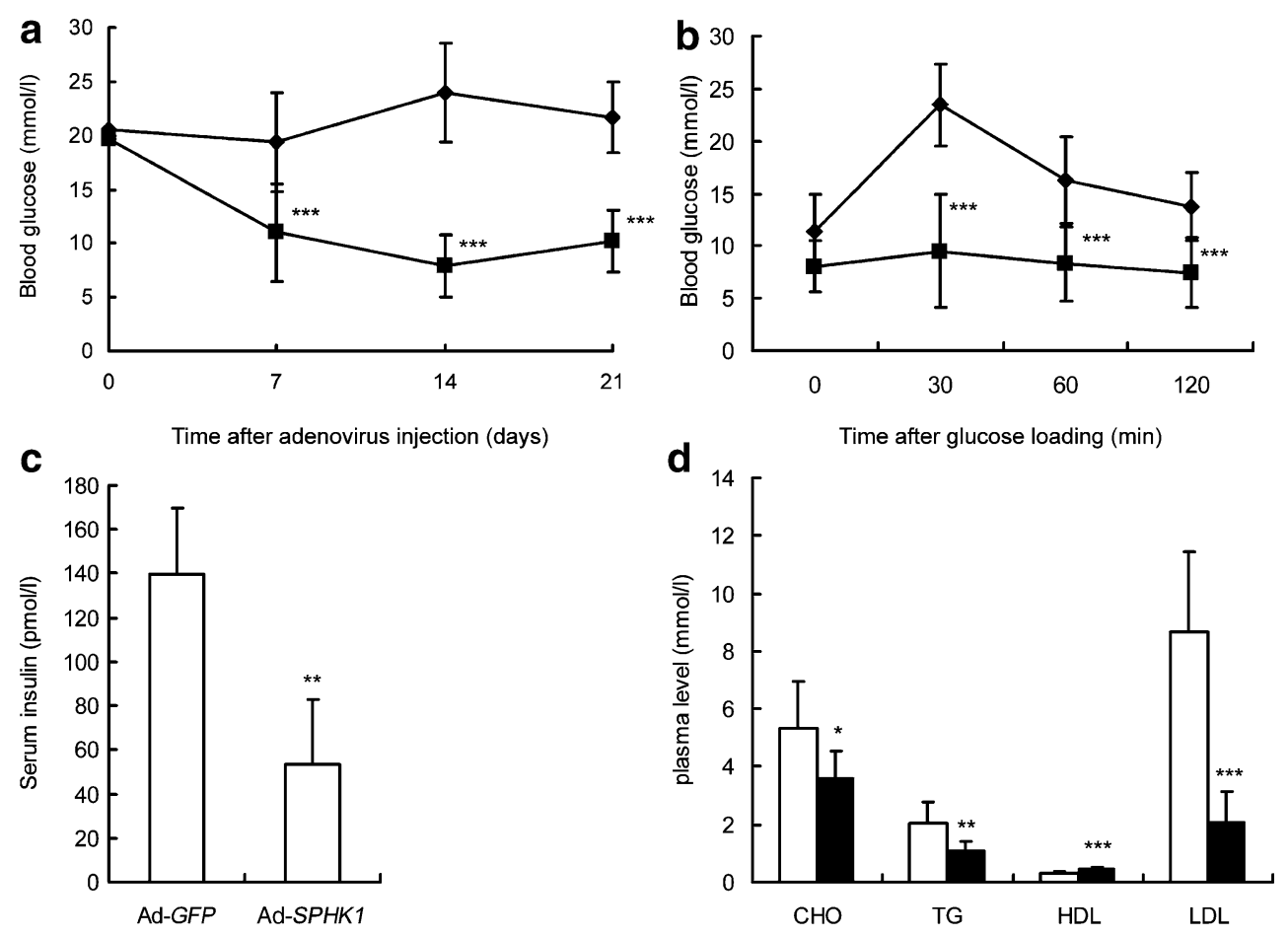

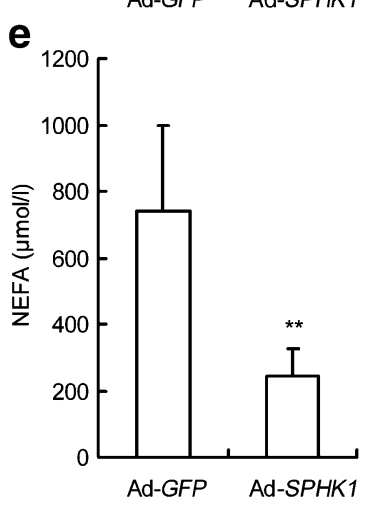

metabolism was also evaluated. Significantly, high serum triacylglycerol, cholesterol and LDL levels were decreased, and HDL was increased in Ad-SPHK1 injected mice (Fig. 2e). All these findings suggest that SPHK1 has a potent hypoglycaemic effect and can improve lipid profiles in type 2 diabetic mice. We further investigated the in vivo effects of SPHK1 on blood glucose levels of normal nondiabetic mice. As shown in ESM Fig. 3, i.v. injection of Ad-SPHK1 had no effect on blood glucose levels and oral glucose tolerance of normal animals. The results indicate that adenovirus-mediated SPHK1 gene transfer might provide a novel and promising strategy to treat type 2 diabetes mellitus.

Effects of SPHK1 gene delivery on liver function and glycogen synthesis in KK/Ay diabetic mice Hepatic insulin resistance is an important pathophysiological feature of type 2 diabetes mellitus and the metabolic syndrome. Previous studies in rodents indicated that systemic infusion of recombinant adenovirus resulted in a preferential target- ing of the transgene to the liver [31]. We then evaluated the metabolic and functional effects of SPHK1 on livers of diabetic mice. Liver weights of mice injected with AdSPHK1 were substantially decreased (Fig. 3a, ESM Table 1). Microscopically, the livers of Ad-SPHK1 injected mice showed markedly reduced steatosis compared with the Ad-GFP control mice (Fig. 3b). Further metabolic measurements showed that SPHK1 gene delivery caused a marked increase in hepatic glycogen content and a significant reduction in the hepatic triacylglycerol content (Fig. 3c,d). Liver enzymes such as AST and ALT were reduced in Ad-SPHK1-injected mice compared with AdGFP control mice (Fig. 3e). Next, we investigated hepatic insulin-signalling proteins. Phosphorylation of molecules involved in insulin signalling such as Akt and GSK3B were enhanced (Fig. 3f) when hepatic SPHK1 activity was increased in Ad-SPHK1-infected mice (ESM Fig. 4). These results indicate that $S P H K 1$ gene transfer resulted in activation of Akt, inactivation of GSK3B, and then an increased glycogen accumulation. 

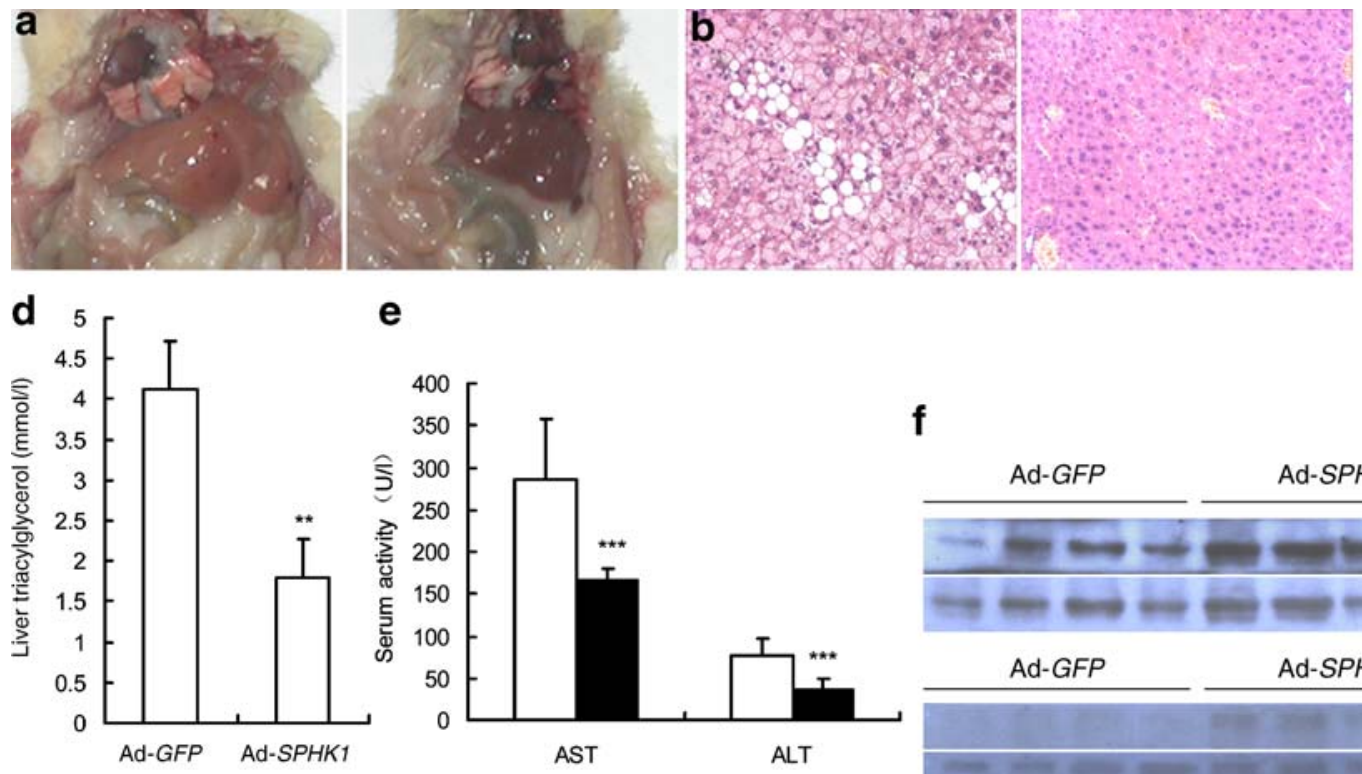

e
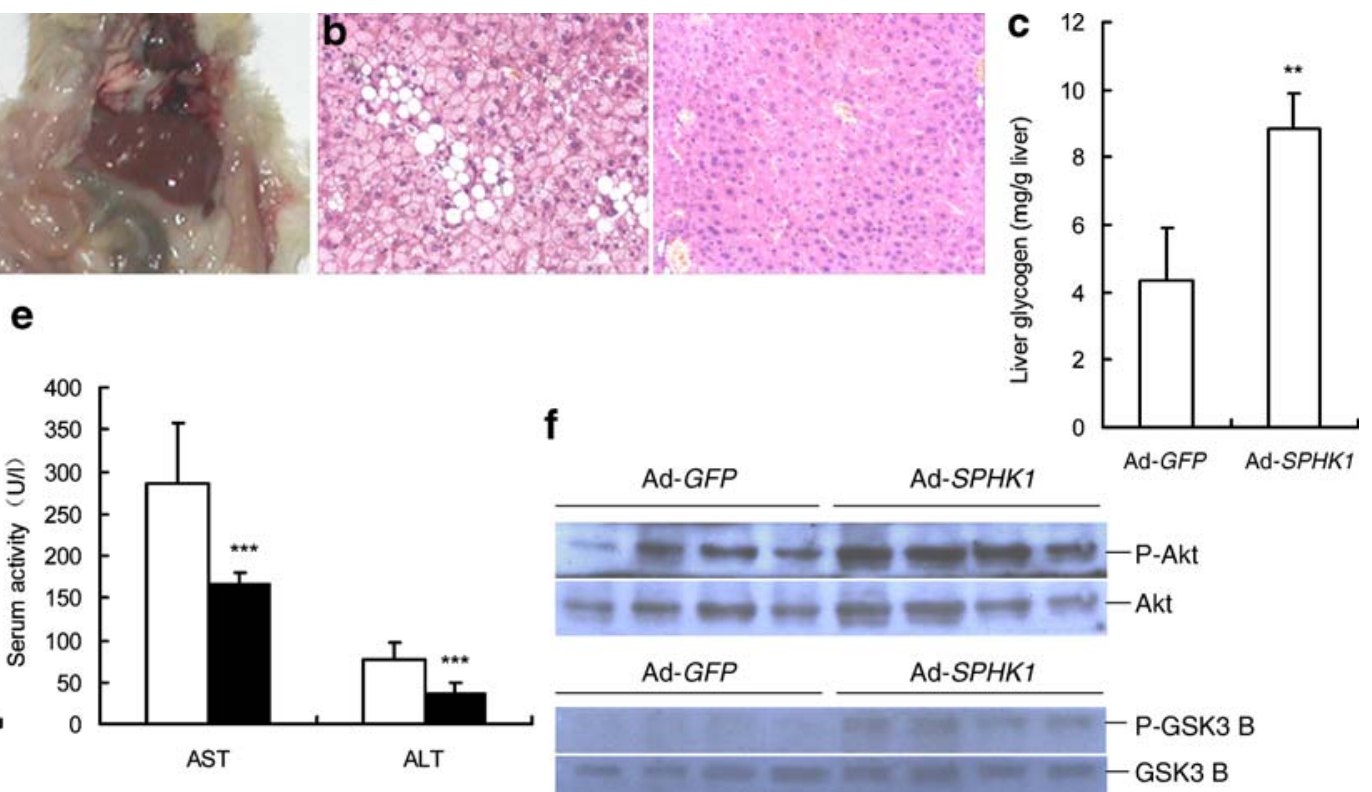

f

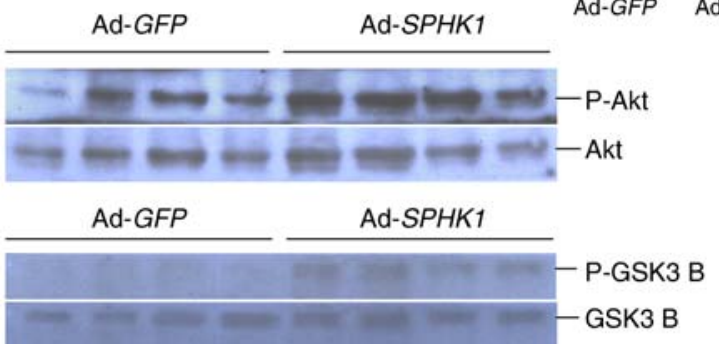

Fig. 3 SPHK1 gene delivery decreases liver steatosis and improves hepatic function in $\mathrm{KK} / \mathrm{Ay}$ diabetic mice. a Livers of $\mathrm{KK} / \mathrm{Ay}$ mice injected with Ad-GFP (left) and Ad-SPHK1 (right). b Histological analysis of livers of KK/Ay mice injected with $\mathrm{Ad}-G F P$ (left) and Ad-SPHK1 (right). c, d Hepatic concentrations of glycogen and triacylglycerol from mice infected with $\mathrm{Ad}-G F P$ or $\mathrm{Ad}-S P H K 1$ at the

endpoint of the study. e Serum AST and ALT activities in Ad-GFP- or Ad-SPHK1-injected animals at the endpoint of the test. Open bars GFP, black bars SPHK1. f Protein levels of insulin-signalling molecules examined by immunoblotting in livers of KK/Ay mice infected with $\mathrm{Ad}-G F P$ or $\mathrm{Ad}-S P H K 1$. Data are means \pm SEM $(n=10)$. $* * p<0.01, * * * p<0.001$ vs GFP group. $P$, Phosphorylated

SPHK1 gene transfer prevents diabetes-induced cardiac injury in KK/Ay diabetic mice To determine whether i.v. injection of adenovirus encoding SPHK1 protects against hyperglycaemia-induced cardiac myocyte injury, the serum levels of LDH and CK were assayed at the endpoint of the study. As shown in Fig. 4a, total LDH and CK activities in the serum of Ad-SPHK1-injected mice were significantly decreased. By means of electron microscopy, we found that significant ultrastructural differences existed in the heart between Ad-GFP- and Ad-SPHK1-treated animals (Fig. $4 \mathrm{~b}, \mathrm{c}$ ). The hearts of mice in the GFP group displayed an obviously disorganised array of the myocardial structure, myofibrillar discontinuation and universal mitochondrial damage (swelling and disrupted cristae). The myofibrillar disruption and mitochondrial damage were significantly prevented in the heart of Ad-SPHK1-infected mice. In addition, only the heart of mice in the GFP group showed many lipid droplets (yellow triangle) and glycogen particles (black triangle) around or inside mitochondria.

SPHK1 gene transfer prevents diabetes-induced renal structural changes in KK/Ay diabetic mice Diabetic nephropathy is one of the most common and lethal complications of diabetes mellitus. In general, diabetic nephropathy advances from the glomerular hyperfiltration stage to the glomerular sclerotic stage with reduced glomerular filtration. It has been proposed that SPHK1/ S1P contributes to the early stages of diabetic nephropathy

as diabetes enhances SPHK1 activity, which results in an increased mesangial cell proliferation, a key event in pathogenesis of the disease $[32,33]$. To determine whether Ad-SPHK1 infection could exacerbate the diabetes-induced renal structural damage or not, we observed the renal structural changes in both groups. Renal glomeruli were obviously small in Ad-SPHK1-injected mice compared with Ad-GFP control mice (Fig. 5a). Under the transmission electron microscope, characteristic diabetic lesions, such as remarkable thickening of glomerular basement membrane, extensive fusion and detachment of podocyte foot processes (solid arrow), were observed in the GFP group, but not in the SPHK1 group (Fig. 5b). These observations indicated that diabetes-enhanced renal SPHK1 activity at the early stage of diabetic nephropathy could not be the cause of the disease. On the contrary, the increase of SPHK1 activity may be a spontaneous and protective response to diabetic nephropathy and the persistent increase in SPHK1 activity (Ad-SPHK1 transfer) can even decrease the incidence of diabetic nephropathy.

\section{Discussion}

Research has indicated that the metabolites of lipids can act as important signal transduction molecules. Sphingolipids, in particular, have emerged as key components of various 

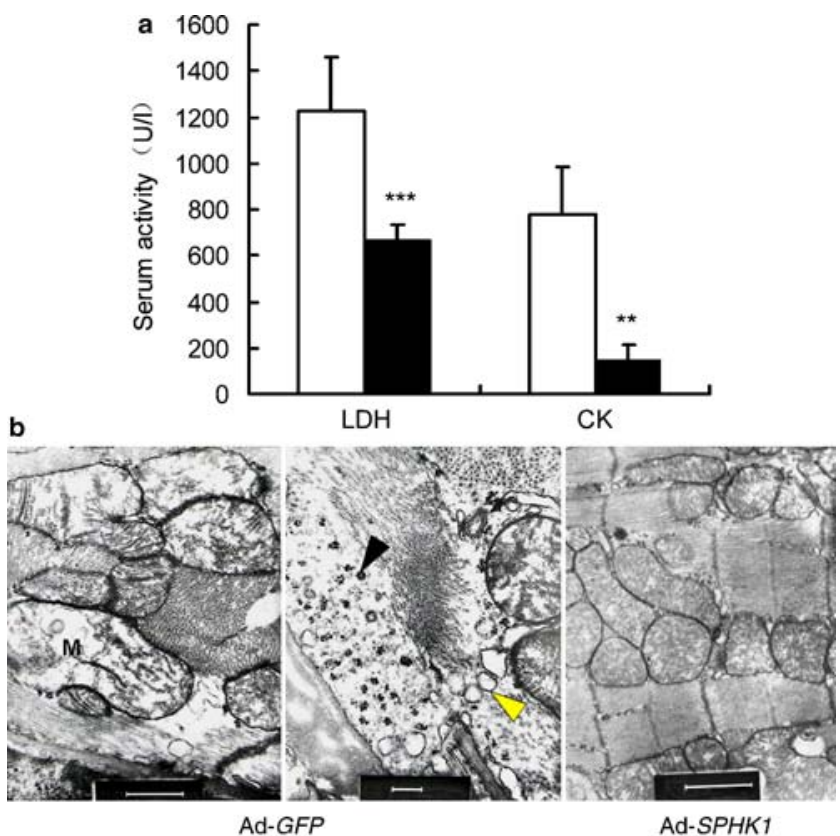

c

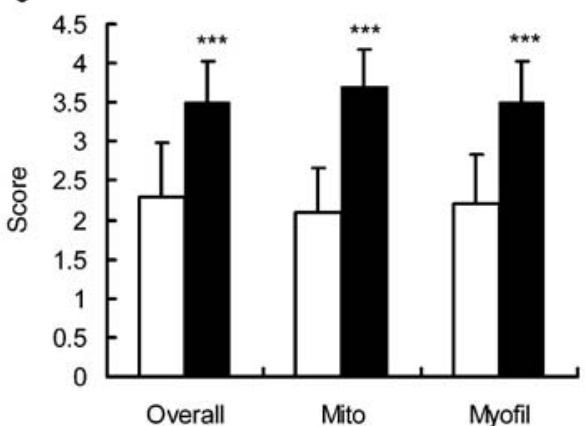

Fig. 4 SPHK1 gene transfer prevents diabetes-induced cardiac ultrastructural changes and serum LDH and CK increase. a Serum LDH and CK activities of KK/Ay mice injected with adenovirus encoding GFP (open bars) or SPHK1 (black bars) at the endpoint of the test. b Cardiac ultrastructures of mice injected i.v. with adenovirus encoding GFP or SPHK1. Only the heart of mice in the GFP group showed many lipid droplets (yellow arrowhead) and glycogen particles (black arrowhead) around or inside mitochondria. $M$ Mitochondria. The white bar indicates $500 \mathrm{~nm}, 200 \mathrm{~nm}$ and $1 \mu \mathrm{m}$ in the panels from left to right, respectively. c Quantification of cardiac morphology. Two blind observers rated ultrastructural features of myocardial tissues in 10-15 randomly selected electron micrographs from each group $(n=3)$. The parameters used for the overall ratings and ratings for mitochondria (Mito) and myofilaments (Myofil) have been described previously [30]. Data are means \pm SEM $(n=10)$. ${ }^{* *} p<0.01, * * * p<0.001$ vs GFP group

cellular responses. SPHK1 is a key enzyme in the sphingolipid metabolic pathway. Accumulated evidence suggests that SPHK1 is implicated in a variety of important biological processes, such as inflammation, atherosclerosis and angiogenesis $[28,34,35]$. In this study, we investigated the potential role of SPHK1 in glucose metabolism and homeostasis. The in vitro study showed that SPHK1 is

\section{a}

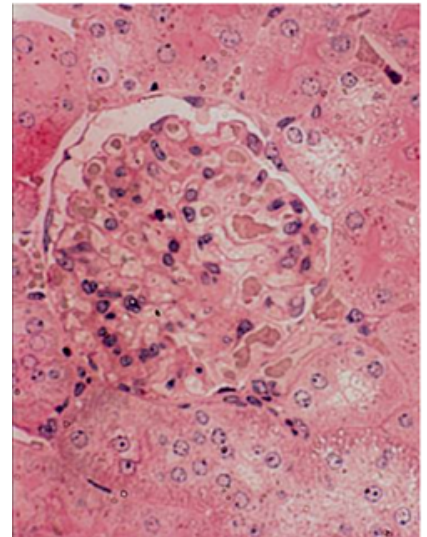

Ad-GFP

b

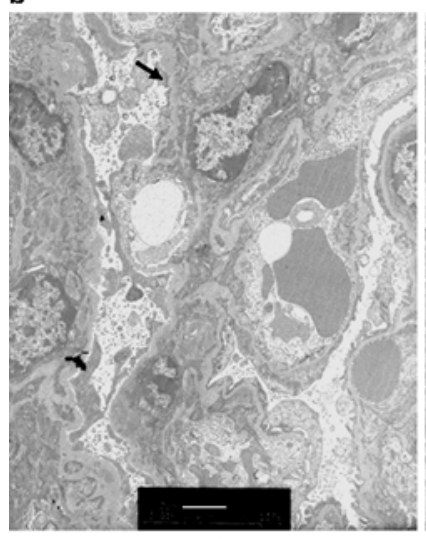

Ad-GFP

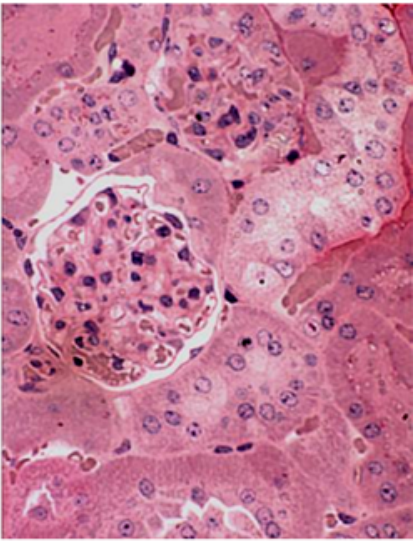

Ad-SPHK1

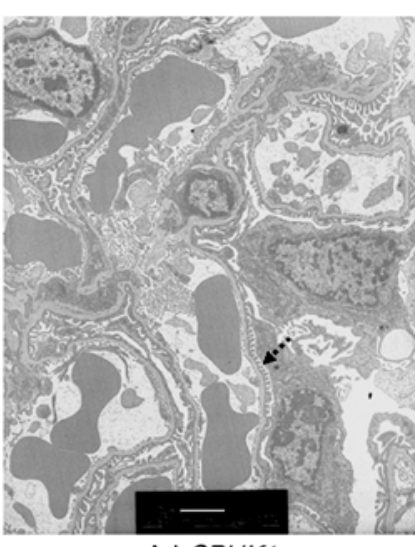

Ad-SPHK1
Fig. $5 S P H K 1$ gene delivery prevents diabetes-induced renal structural changes. a Renal structures of mice injected i.v. with Ad-GFP or Ad-SPHK1. Enlarged glomerulus and thickened basal membrane in mice of the GFP group are shown. b Renal ultrastructures of mice injected with Ad-GFP or Ad-SPHK1. Characteristic diabetic lesions, such as remarkable thickening of glomerular basement membrane, extensive fusion and detachment of podocyte foot processes (solid arrow), were observed in the GFP group, but not in the SPHK1 group (dotted arrow). The white bar indicates $2 \mu \mathrm{m}$

implicated in the insulin-signalling pathway and plays a role in insulin-stimulated glucose uptake by both 7721 hepatoma cells and $\mathrm{C} 2 \mathrm{C} 12$ myotube cells. In vivo studies indicated that SPHK1 gene delivery markedly reduced blood glucose level and greatly improved lipid profiles in $\mathrm{KK} /$ Ay diabetic mice. Interestingly, the blood glucose level in normal, non-diabetic animals was not affected by $\mathrm{Ad}-$ SPHK1 administration. These results show that SPHK1 would be another important lipid kinase involved in glucose metabolism, and that adenovirus-mediated SPHK1 gene transfer would be a promising strategy to treat type 2 diabetes mellitus.

Insulin-stimulated glucose uptake and utilisation by peripheral tissues, such as liver, muscle and fat, is crucial for maintaining normal blood glucose level. We first 
examined the role of SPHK1 in basal and insulin-stimulated glucose uptake in vitro. SPHK1 overexpression led to a marked increase in basal glucose uptake and an enhancement in insulin-stimulated glucose uptake as well, whether in 7721 cells or $\mathrm{C} 2 \mathrm{C} 12$ cells. Consistent with this, glycogen contents in liver and muscle (data not shown) of AdSPHK1-treated diabetic mice were also significantly increased. These results suggest that stimulating glucose uptake in peripheral tissues might be a major cause of the hypoglycaemic effect of SPHK1. Surprisingly, DMS, a potent SPHK1 inhibitor, completely abrogated insulin effects. This inhibitory effect of DMS on insulin signalling can not only be explained by its blockage of SPHK1 activity, because DMS has also been shown to inhibit protein kinase $\mathrm{C}$ [36] and to affect muscarinic M3 responses by mechanisms other than sphingosine kinase inhibition [37]. In a recent study, it was shown that insulin has no effect on SPHK1 enzyme activity in vitro in vascular endothelial cells [38]. We showed here that insulin could enhance SPHK1 activity in both 7721 and $\mathrm{C} 2 \mathrm{C} 12$ cell lines. The results indicate that insulin may activate SPHK1 in a cell-specific manner.

Type 2 diabetes mellitus is characterised by an impaired insulin sensitivity and resultant dysregulation of glucose and lipid metabolism. KK/Ay mice exhibit morbid obesity and metabolic abnormalities such as hyperglycaemia, glucose intolerance and hyperinsulinaemia, and are known to serve as an excellent model of type 2 diabetes mellitus [39]. The high serum insulin levels of KK/Ay mice, which are a result of insulin resistance, were markedly lowered by administering Ad-SPHK1, and so were high serum NEFA levels, which are a major cause of insulin resistance. These findings suggest that decreasing the insulin resistance by SPHK1 would be a major mechanism for its hypoglycaemic activity, and if insulin is involved in the action of SPHK1 in vivo it induces an increase in insulin sensitivity rather than insulin release. Because hepatic steatosis in patients with hypertriglycerolaemia is linked to a decrease in insulin sensitivity [40], the insulin-sensitising effect of SPHK1 was further supported by the following results: Ad-SPHK1 injection activated the insulin-signalling kinases such as Akt and GSK3B in livers, alleviated the fatty liver, and reduced the serum triacylglycerol and cholesterol. Currently, the mechanism of action of SPHK1 in lipid metabolism and management is still unclear and needs to be further explored.

It is of interest to note that the body weight of $\mathrm{Ad}-$ SPHK1-treated mice was markedly decreased (ESM Table 1). It is well known that insulin resistance is very often accompanied by obesity. Obesity not only increases the chance of developing type 2 diabetes, but is also associated with insulin resistance and other morbidity [41]. In obese patients with type 2 diabetes, insulin resistance is significantly worse than that in non-obese diabetic individuals [42]. Other studies have shown that insulin sensitivity in type 2 diabetes patients improved with weight loss [43]. We indicated here that Ad-SPHK1 infection alleviated the fatty livers and reduced the fat and glycogen accumulation in the myocardium of KK/Ay diabetic mice. Thus, Ad-SPHK1 would be particularly beneficial for treatment of diabetes, especially obese diabetic patients, and improving glucose utilisation and reducing fat deposition in peripheral tissues might be potential mechanisms of the effects of Ad-SPHK1 infection on body weight. In a recent study, we showed that SHP-2 tyrosine phosphatase interacts with SPHK1 directly and plays a crucial role in regulating the basal and hepatocyte growth factor-stimulated SPHK1 activity [44]. Interestingly, a prominent phenotype of the Shp2 mutant mice was the development of early-onset obesity, with increased serum levels of leptin, insulin, glucose and triacylglycerol [45]. These results thereby indicate that SPHK1 might be an important molecule in the control of body weight and energy metabolism.

Diabetes mellitus of long duration is associated with several complications, such as diabetic myocardiopathy, diabetic neuropathy and nephropathy. Increased accumulation of glycogen in the myocardium is a typical feature in diabetic animal models [46], and can finally lead to diabetic cardiomyopathy. As shown in Fig. 4b, there are many lipid droplets and glycogen particles in the hearts of Ad-GFPinfected KK/Ay diabetic mice but not in Ad-SPHK1treated animals; serum LDH and CK enzymatic activity in Ad-SPHK1-treated mice were also markedly reduced. These results showed that SPHK1 plays a potential role in overcoming the impairment in glycogenolysis, improving the use of myocardial glycogen and preserving the heart function. With diabetic patients often having impaired cardiac function and heart failure, these data suggest a new role for SPHK1 in this debilitating disease. Moreover, the whole diabetic phenotype of liver and kidney was markedly reversed in Ad-SPHK1-treated mice. Except for improving the blood glucose level by Ad-SPHK1 injection, other possible explanations for this result are as follows: (1) SPHK1, as a survival factor, might protect cardiomyocytes, hepatocytes and renal cells against hyperglycaemia-induced death; (2) SPHK1 might have a potential anti-inflammatory function, for a recent report showed that S1P acts as an immunosuppressant by modifying lymphocytic migration [47]; and (3) SPHK1/S1P is involved in the regulation of vascular tone and could increase the resistance of endothelial cells and enhance barrier integrity [48]. Whether the above potential mechanisms are involved in the recovery of the diabetic phenotype by SPHK1 gene delivery needs to be further verified.

In conclusion, regardless of the above-mentioned multiple mechanisms for SPHK1 action, our findings suggest that SPHK1 is a novel molecule in the regulation of glucose 
and fat metabolism and may be used as a favourable therapeutic target of type 2 diabetes mellitus.

Acknowledgements This project was supported by Chinese National Basic Research and Development Grants '973' (no. 2004CB518801; no. 2006CB504105) and Chinese National Science Foundation (no. 30500208). We also thank Z. X. Tang and J. X. Wang for amending this manuscript.

Duality of interest There is no duality of interest for any authors listed.

\section{References}

1. Choo HJ, Kim JH, Kwon OB et al (2006) Mitochondria are impaired in the adipocytes of type 2 diabetic mice. Diabetologia 49:784-791

2. Lin Y, Itani SI, Kurowski TG et al (2001) Inhibition of insulin signaling and glycogen synthesis by phorbol dibutyrate in rat skeletal muscle. Am J Physiol Endocrinol Metab 281:E8-E15

3. Dandona P, Aljada A, Bandyopadhyay A (2004) Inflammation: the link between insulin resistance, obesity and diabetes. Trends Immunol 25:4-7

4. Hotamisligil GS (2003) Inflammatory pathways and insulin action. Int J Obes Relat Metab Disord 27(Suppl 3):S53-S55

5. Boden G, She P, Mozzoli M et al (2005) Free fatty acids produce insulin resistance and activate the proinflammatory nuclear factorKB pathway in rat liver. Diabetes 54:3458-3465

6. Armstrong KA, Hiremagalur B, Haluska BA et al (2005) Free fatty acids are associated with obesity, insulin resistance, and atherosclerosis in renal transplant recipients. Transplantation 80:937-944

7. Brehm A, Krssak M, Schmid AI, Nowotny P, Waldhausl W, Roden M (2006) Increased lipid availability impairs insulinstimulated ATP synthesis in human skeletal muscle. Diabetes 55:136-140

8. Summers SA (2006) Ceramides in insulin resistance and lipotoxicity. Prog Lipid Res 45:42-72

9. Summers SA, Nelson DH (2005) A role for sphingolipids in producing the common features of type 2 diabetes, metabolic syndrome X, and Cushing's syndrome. Diabetes 54:591-602

10. Dressler KA, Mathias S, Kolesnick RN (1992) Tumor necrosis factor-alpha activates the sphingomyelin signal transduction pathway in a cell-free system. Science 255:1715-1718

11. Summers SA, Garza LA, Zhou H, Birnbaum MJ (1998) Regulation of insulin-stimulated glucose transporter GLUT4 translocation and Akt kinase activity by ceramide. Mol Cell Biol 18:5457-5464

12. Schmitz-Peiffer C, Craig DL, Biden TJ (1999) Ceramide generation is sufficient to account for the inhibition of the insulin-stimulated PKB pathway in $\mathrm{C} 2 \mathrm{C} 12$ skeletal muscle cells pretreated with palmitate. J Biol Chem 274:24202-24210

13. Chavez JA, Knotts TA, Wang LP et al (2003) A role for ceramide, but not diacylglycerol, in the antagonism of insulin signal transduction by saturated fatty acids. J Biol Chem 278:10297-10303

14. Teruel T, Hernandez R, Lorenzo M (2001) Ceramide mediates insulin resistance by tumor necrosis factor-alpha in brown adipocytes by maintaining Akt in an inactive dephosphorylated state. Diabetes 50:2563-2571

15. Shepherd PR, Withers DJ, Siddle K (1998) Phosphoinositide 3kinase: the key switch mechanism in insulin signaling. Biochem $\mathrm{J}$ 333:471-490
16. Cantley LC (2002) The phosphoinositide 3-kinase pathway. Science 296:1655-1657

17. Taniguchi CM, Emanuelli B, Kahn CR (2006) Critical nodes in signaling pathways: insights into insulin action. Nat Rev Mol Cell Biol 7:85-96

18. Hannun YA, Luberto C, Argraves KM (2001) Enzymes of sphingolipid metabolism: from modular to integrative signaling. Biochemistry 40:4893-4903

19. Merrill AH Jr, Schmelz EM, Dillehay DL et al (1997) Sphingolipids - the enigmatic lipid class: biochemistry, physiology, and pathophysiology. Toxicol Appl Pharmacol 142:208-225

20. Maceyka M, Payne SG, Milstien S, Spiegel S (2002) Sphingosine kinase, sphingosine-1-phosphate, and apoptosis. Biochim Biophys Acta 1585:193-201

21. Taha TA, Hannun YA, Obeid LM (2006) Sphingosine kinase: biochemical and cellular regulation and role in disease. J Biochem Mol Biol 39:113-131

22. Spiegel S, Kolesnick R (2002) Sphingosine 1-phosphate as a therapeutic agent. Leukemia 16:1596-1602

23. Obeid LM, Linardic CM, Karolak LA, Hannun YA (1993) Programmed cell death induced by ceramide. Science 259:1769-1771

24. Cuvillier O, Pirianov G, Kleuser B, Vanek PG, Coso OA, Gutkind S, Spiegel S (1996) Suppression of ceramide-mediated programmed cell death by sphingosine-1-phosphate. Nature 381: $800-803$

25. Verma NK, Singh J, Dey CS (2004) PPAR-gamma expression modulates insulin sensitivity in $\mathrm{C} 2 \mathrm{C} 12$ skeletal muscle cells. Br J Pharmacol 143:1006-1013

26. Olivera A, Kohama T, Tu Z, Milstien S, Spiegel S (1998) Purification and characterization of rat kidney sphingosine kinase. J Biol Chem 273:12576-12583

27. Edsall LC, Spiegel S (1999) Enzymatic measurement of sphingosine 1-phosphate. Anal Biochem 272:80-86

28. Ancellin N, Colmont C, Su J et al (2002) Extracellular export of sphingosine kinase-1 enzyme. Sphingosine 1-phosphate generation and the induction of angiogenic vascular maturation. J Biol Chem 277:6667-6675

29. Yahagi N, Shimano H, Hasty AH et al (2002) Absence of sterol regulatory element-binding protein-1 (SREBP-1) ameliorates fatty livers but not obesity or insulin resistance in $\operatorname{Lep}(\mathrm{ob}) / \mathrm{Lep}(\mathrm{ob})$ mice. J Biol Chem 277:19353-19357

30. Liang Q, Carlson EC, Donthi RV, Kralik PM, Shen X, Epstein PN (2002) Overexpression of metallothionein reduces diabetic cardiomyopathy. Diabetes 51:174-181

31. Nakagawa Y, Shimano H, Yoshikawa $T$ et al (2006) TFE3 transcriptionally activates hepatic IRS-2, participates in insulin signaling and ameliorates diabetes. Nat Med 12:107-113

32. Geoffroy K, Troncy L, Wiernsperger N, Lagarde M, El Bawab S (2005) Glomerular proliferation during early stages of diabetic nephropathy is associated with local increase of sphingosine-1phosphate levels. FEBS Lett 579:1249-1254

33. Katsuma S, Hada Y, Ueda T et al (2002) Signaling mechanisms in sphingosine 1-phosphate-promoted mesangial cell proliferation. Genes Cells 7:1217-1230

34. Xu CB, Hansen-Schwartz J, Edvinsson L (2004) Sphingosine signaling and atherogenesis. Acta Pharmacol Sin 25:849-854

35. Jolly PS, Bektas M, Watterson KR et al (2005) Expression of SPHK1 impairs degranulation and motility of RBL-2H3 mast cells by desensitizing S1P receptors. Blood 105:4736-4742

36. Khan WA, Dobrowsky R, el Touny S, Hannun YA (1990) Protein kinase $\mathrm{C}$ and platelet inhibition by D-erythrosphingosine: comparison with $N, N$-dimethylsphingosine and commercial preparation. Biochem Biophys Res Commun 172:683-691

37. Young KW, Channing DR, Nahorski SR (2000) Effect of dimethylsphingosine on muscarinic $\mathrm{M}(3)$ receptor signaling in SH-SY5Y cells. Eur J Pharmacol 402:55-59 
38. Wang LJ, Xing XP, Holmes A (2005) Activation of the sphingosine kinase-signaling pathway by high glucose mediates the proinflammatory phenotype of endothelial cells. Circ Res 97:891-899

39. Iwatsuka H, Shino A, Suzuoki Z (1970) General survey of diabetic features of yellow KK mice. Endocrinol Jpn 17:23-35

40. Maruhama Y, Ohneda A, Tadaki H et al (1975) Hepatic steatosis and the elevated plasma insulin level in patients with endogenous hypertriglyceridemia. Metabolism 24:653-664

41. Kruszynska YT, Olefsky JM (1996) Cellular and molecular mechanisms of non-insulin dependent diabetes mellitus. J Investig Med 44:413-428

42. Seely BL, Olefsky JM (1993) Potential cellular and genetic mechanisms for insulin resistance in common disorders of obesity and diabetes. In: Moller D (ed) Insulin resistance and its clinical disorders. Wiley, Chichester, pp 187-252

43. DeFronzo RA, Ferrannini E (1991) Insulin resistance. A multifaceted syndrome responsible for NIDDM, obesity, hypertension, dyslipidemia, and atherosclerotic cardiovascular disease. Diabetes Care 14:173-194

44. Duan HF, Qu CK, Zhang QW et al (2006) Shp-2 tyrosine phosphatase is required for hepatocyte growth factor-induced activation of sphingosine kinase and migration in embryonic fibroblasts. Cell Signal 18:2049-2055

45. Zhang EE, Chapeau E, Hagihara K, Feng GS (2004) Neuronal Shp2 tyrosine phosphatase controls energy balance and metabolism. Proc Natl Acad Sci U S A 101:16064-16069

46. Penpargkul S, Schaible T, Yipintsoi T, Scheuer J (1980) The effect of diabetes on performance and metabolism of rat hearts. Circ Res 47:911-921

47. Massberg S, von Andrian UH (2006) Fingolimod and sphingosine-1-phosphate-modifiers of lymphocyte migration. N Engl J Med 355:1088-1091

48. Bolz SS, Vogel L, Sollinger D et al (2003) Sphingosine kinase modulates microvascular tone and myogenic responses through activation of RhoA/Rho kinase. Circulation 108:342-347 